PROCEEDINGS OF THE

AMERICAN MATHEMATICAL SOCIETY

Volume 129, Number 6, Pages 1739-1744

S 0002-9939(00)05751-8

Article electronically published on October 31, 2000

\title{
CONVEX CURVES, RADON TRANSFORMS \\ AND CONVOLUTION OPERATORS DEFINED BY SINGULAR MEASURES
}

\author{
FULVIO RICCI AND GIANCARLO TRAVAGLINI \\ (Communicated by Christopher D. Sogge)
}

\begin{abstract}
Let $\Gamma$ be a convex curve in the plane and let $\mu \in M\left(\mathbb{R}^{2}\right)$ be the arc-length measure of $\Gamma$. Let us rotate $\Gamma$ by an angle $\theta$ and let $\mu_{\theta}$ be the corresponding measure. Let $T f(x, \theta)=f * \mu_{\theta}(x)$. Then

$$
\|T f\|_{L^{3}\left(\mathbb{T} \times \mathbb{R}^{2}\right)} \leq c\|f\|_{L^{3 / 2}\left(\mathbb{R}^{2}\right)} .
$$

This is optimal for an arbitrary $\Gamma$. Depending on the curvature of $\Gamma$, this estimate can be improved by introducing mixed-norm estimates of the form

$$
\|T f\|_{L^{s}\left(\mathbb{T}, L^{p^{\prime}}\left(\mathbb{R}^{2}\right)\right)} \leq c\|f\|_{L^{p}\left(\mathbb{R}^{2}\right)}
$$

where $p$ and $p^{\prime}$ are conjugate exponents.
\end{abstract}

\section{Introduction AND STATEMENTS OF THE RESUlts}

Let $\Gamma:[a, b] \rightarrow \mathbb{R}^{2}$ be a convex planar curve (i.e. it locally coincides with the graph of a convex function in suitable coordinates). Let $\mu^{\Gamma}=\mu$ be the measure supported on $\Gamma$ induced by Lebesgue measure on $\mathbb{R}^{2}$. Under appropriate curvature assumptions on $\Gamma, \mu$ is known to be $L^{p}$ improving, i.e. $\|f * \mu\|_{L^{q}\left(\mathbb{R}^{2}\right)} \leq c\|f\|_{L^{p}\left(\mathbb{R}^{2}\right)}$ for some $q>p$.

Restricting our attention to the case $q=p^{\prime}$, it is known that the best possible result is an $L^{3 / 2}-L^{3}$ estimate, and that this is attained when $\Gamma$ has non-vanishing curvature at every point (see e.g. $[\underline{\mathrm{S}}]$ or $[\mathrm{O}]$ ):

$$
\left\|f * \mu^{\Gamma}\right\|_{L^{3}\left(\mathbb{R}^{2}\right)} \leq c\|f\|_{L^{3 / 2}\left(\mathbb{R}^{2}\right)} .
$$

Weaker $L^{p}-L^{p^{\prime}}$ estimates, for certain $p<2$, still hold if the curvature vanishes of finite order at some isolated point $\left[\mathrm{RS}\right.$. At the other extreme, no $L^{p}$-improving occurs if $\Gamma$ contains a segment (this is due to the fact that if $f$ is infinite on a segment $S$, then its convolution with $\mu^{S}$ remains infinite of the same order).

A different form of $L^{p}$-improving connected with integration over straight lines is given by the mapping properties of the Radon transform. Replacing integration over straight lines by integration over segments, the main result in OS] says that

Received by the editors May 15, 1999 and, in revised form, September 27, 1999.

2000 Mathematics Subject Classification. Primary 42B10.

Key words and phrases. Convolution operators, singular measures, Radon transforms.

(C)2000 American Mathematical Society 
if

$$
\mathcal{R} f(t, \theta)=\int_{|s|<1} f\left(\sigma_{\theta}(t, s)\right) d s
$$

(here $t \in \mathbb{R}$ and $\sigma_{\theta}$ denotes rotation by $\theta \in \mathbb{T}$ ), then

$$
\|\mathcal{R} f\|_{L^{3}(\mathbb{T} \times \mathbb{R})} \leq c\|f\|_{L^{3 / 2}\left(\mathbb{R}^{2}\right)} .
$$

A different formulation of (1.2) can be given as follows. Let $\mu$ denote the arclength measure on a given segment in the plane, and let $\mu_{\theta}$ be the arc-length measure on the same segment rotated by an angle $\theta \in \mathbb{T}$. Then, given $f \in L^{3 / 2}\left(\mathbb{R}^{2}\right)$, the function $f * \mu_{\theta}$ is in $L^{3}\left(\mathbb{R}^{2}\right)$ for almost every $\theta \in \mathbb{T}$, and $\left\|f * \mu_{\theta}\right\|_{L^{3}\left(\mathbb{R}^{2}\right)} \in L^{3}(\mathbb{T})$.

Our first result says that the same holds for general convex curves.

Theorem 1. Let $\Gamma$ be a convex curve and let $\mu$ be the measure on $\Gamma$ induced by Lebesgue measure on $\mathbb{R}^{2}$. Let $\mu_{\theta}^{\Gamma}=\mu_{\theta}$ be the measure obtained from $\mu$ by composition with the rotation $\sigma_{\theta}$ by an angle $\theta \in[-\pi, \pi]$. Then

$$
\left\{\int_{-\pi}^{\pi} \int_{\mathbb{R}^{2}}\left|\left(f * \mu_{\theta}\right)(x)\right|^{3} d x d \theta\right\}^{1 / 3} \leq c\left\{\int_{\mathbb{R}^{2}}|f(t)|^{3 / 2} d t\right\}^{2 / 3} .
$$

The estimate (1.3) is clearly weaker than (1.1). The latter says in fact that $\left\|f * \mu_{\theta}^{\Gamma}\right\|_{L^{3}\left(\mathbb{R}^{2}\right)} \in L^{\infty}(\mathbb{T})$ when $\Gamma$ has non-vanishing curvature and $f \in L^{3 / 2}\left(\mathbb{R}^{2}\right)$.

The study of $L^{p}$-improving estimates requires the knowledge of the decay at infinity of the Fourier transform of the given measure. The optimal estimate (1.1) follows from the uniform estimate

$$
\left|\hat{\mu}^{\Gamma}(\xi)\right| \leq c|\xi|^{-1 / 2}
$$

which holds, for $|\xi|$ large, when $\Gamma$ has non-zero curvature.

What we use instead is the decay of the $L^{2}$ averages of $\hat{\mu}^{\Gamma}$ over the circles $|\xi|=r$. A result of A.N. Podkorytov says that these $L^{2}$ averages decay like $r^{-1 / 2}$ for general convex curves (see $[\mathrm{P}]$ or $[\mathrm{BIT}]$, and [Ra] for an earlier result for analytic curves).

We next discuss the possibility of improving (1.3) under appropriate assumptions on the curvature of $\Gamma$. In order to do so, we introduce the class of mixed-norm spaces $L^{s}\left(\mathbb{T}, L^{q}\left(\mathbb{R}^{2}\right)\right)$.

If $T$, or $T^{\Gamma}$, denotes the operator $T f(x, \theta)=f * \mu_{\theta}^{\Gamma}(x)$, we discuss the values of $p$ and $s$ for which the inequality

$$
\left\|T^{\Gamma} f\right\|_{L^{s}\left(\mathbb{T}, L^{\left.p^{\prime}\left(\mathbb{R}^{2}\right)\right)}\right.} \leq c\|f\|_{L^{p}\left(\mathbb{R}^{2}\right)}
$$

holds for every $f \in L^{p}\left(\mathbb{R}^{2}\right)$.

We begin with some simple remarks.

Remark. If the convolution operator $f \rightarrow f * \mu$ is bounded from $L^{p}\left(\mathbb{R}^{2}\right)$ to $L^{p^{\prime}}\left(\mathbb{R}^{2}\right)$, then (1.4) holds for every $s$. Indeed, writing $f_{\theta}(x)=f\left(\sigma_{\theta}(x)\right)$ one has

$$
\left\|f * \mu_{\theta}\right\|_{L^{p^{\prime}\left(\mathbb{R}^{2}\right)}}=\left\|f_{-\theta} * \mu\right\|_{L^{p^{\prime}\left(\mathbb{R}^{2}\right)}} \leq c\left\|f_{\theta}\right\|_{L^{p}\left(\mathbb{R}^{2}\right)}=c\|f\|_{L^{p}\left(\mathbb{R}^{2}\right)} .
$$

Remark. A necessary condition for (1.4) to hold is that $p \geq 3 / 2$. This can be shown by the standard method of testing the operator on the characteristic function of a small disc.

By the above remarks and (1.1) we deduce that the problem is trivial if $\Gamma$ has non-zero curvature at every point. The interesting case is when the curvature of $\Gamma$ vanishes of finite order at some point. We shall restrict our attention to the 
typical examples $\Gamma_{\gamma}(t)=\left(t,|t|^{\gamma}\right)$ with $|t| \leq 1$ and $2<\gamma<\infty$, so that the curvature vanishes of order $\gamma-2$ at $t=0$.

Theorem 2. Let $\Gamma=\Gamma_{\gamma}$ be the graph of the function $x_{2}=\left|x_{1}\right|^{\gamma}$, where $\gamma>2$ and $\left|x_{1}\right| \leq 1$. Let $\mu^{\gamma}=\mu^{\Gamma_{\gamma}}, \mu_{\theta}^{\gamma}=\mu^{\gamma} \circ \sigma_{\theta}$ and $T_{\gamma}=T^{\Gamma_{\gamma}}$. Then

$$
\begin{aligned}
& \left\|T_{\gamma} f\right\|_{L^{\frac{3(\gamma-1)}{\gamma-2}-\delta}\left(\mathbb{T}, L^{3}\left(\mathbb{R}^{2}\right)\right)} \leq c_{\delta}\|f\|_{L^{3 / 2}\left(\mathbb{R}^{2}\right)} \quad \text { for any small } \delta>0,
\end{aligned}
$$

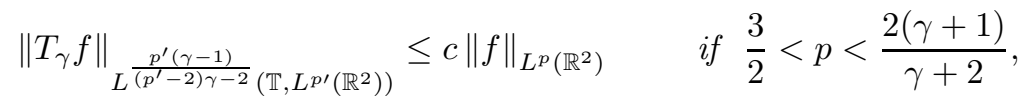

$$
\begin{aligned}
& \left\|T_{\gamma} f\right\|_{L^{\infty}\left(\mathbb{T}, L^{p^{\prime}}\left(\mathbb{R}^{2}\right)\right)} \leq c\|f\|_{L^{p}\left(\mathbb{R}^{2}\right)} \quad \text { if } \frac{2(\gamma+1)}{\gamma+2} \leq p \leq 2 .
\end{aligned}
$$

Moreover,

$$
\begin{aligned}
& \left\|T_{\gamma} f\right\|_{L^{s}\left(\mathbb{T}, L^{3}\left(\mathbb{R}^{2}\right)\right)} \leq c\|f\|_{L^{3 / 2}\left(\mathbb{R}^{2}\right)} \quad \text { implies } s \leq \frac{3(\gamma-1)}{\gamma-2}, \\
& \left\|T_{\gamma} f\right\|_{L^{s}\left(\mathbb{T}, L^{p^{\prime}}\left(\mathbb{R}^{2}\right)\right)} \leq c\|f\|_{L^{p}\left(\mathbb{R}^{2}\right)} \quad \text { and } \frac{3}{2}<p<\frac{2(\gamma+1)}{\gamma+2} \\
& \text { imply } s \leq \frac{p^{\prime}(\gamma-1)}{\left(p^{\prime}-2\right) \gamma-2}, \\
& \left\|T_{\gamma} f\right\|_{L^{\infty}\left(\mathbb{T}, L^{\left.p^{\prime}\left(\mathbb{R}^{2}\right)\right)}\right.} \leq c\|f\|_{L^{p}\left(\mathbb{R}^{2}\right)} \quad \text { implies } p \geq \frac{2(\gamma+1)}{\gamma+2} .
\end{aligned}
$$

The proof of Theorem 2 depends on certain sharp estimates for the $L^{p}$ average decay of $\hat{\mu}^{\Gamma_{\gamma}}$ (compare Lemma 1 and Lemma 2 below).

Remarks. We do not know whether $\delta$ is necessary in (ii). If we could take $\delta=0$, then (ii) would follow from $(i)$ and (iii) by interpolation.

We point out that that (iii) is a particular case of a more general result essentially proved in [C] and [RS] (see [Ri] for an explicit statement).

The case of a segment can be seen as a limit case for $\gamma \rightarrow \infty$. One has

$$
\left\|T^{S} f\right\|_{L^{p^{\prime} /\left(p^{\prime}-2\right)}\left(\mathbb{T}, L^{p^{\prime}}\left(\mathbb{R}^{2}\right)\right)} \leq c\|f\|_{L^{p}\left(\mathbb{R}^{2}\right)}
$$

for $\frac{3}{2} \leq p \leq 2$, and $\frac{p^{\prime}}{p^{\prime}-2}$ cannot be replaced by a smaller exponent [OS].

The same conclusion of Theorem 2 holds if $\Gamma_{\gamma}$ is replaced by a translate of it (the proof requires minor modifications that we shall omit).

\section{Proofs}

The proof of Theorem 1 is based on the following lemma due to A.N. Podkorytov (see [P]) concerning the $L^{2}$ average decay of $\widehat{\mu}$.

Lemma 1. Let $\Gamma$ be a convex curve in the plane, let $\mu=\mu^{\Gamma}$ and $\mu_{\theta}=\mu \circ \sigma_{\theta}$. Then

$$
\int_{-\pi}^{\pi}\left|\widehat{\mu_{\theta}}(\xi)\right|^{2} d \theta \leq c|\xi|^{-1} .
$$

Podkorytov's result depends on the fact that the Fourier transform has the expected decay in most directions and averaging in $L^{p}(p<\infty)$ prevents the bad directions from having too much of an impact. 
Proof of Theorem 1. By decomposing $\Gamma$ into a finite number of arcs, we can assume that $\Gamma$ is the graph of a function of the first variable. Let $z \in \mathbb{C}$ such that $\operatorname{Re} z>0$. Define $i_{z}$ via

$$
\left\langle i_{z}, \varphi\right\rangle=\frac{1}{\Gamma(z)} \int_{0}^{\infty} \varphi(t) t^{z-1} d t
$$

Define $\mu_{\theta}^{z}$ via

$$
\widehat{\mu_{\theta}^{z}}(\xi)=\widehat{\mu_{\theta}}(\xi) \widehat{i_{z}}\left(\left(\sigma_{\theta} \xi\right)_{2}\right)
$$

where $\left(\sigma_{\theta} \xi\right)_{2}$ denotes the second coordinate of the vector $\sigma_{\theta} \xi$. We introduce the analytic family of operators

$$
T^{z}: f \rightarrow f * \mu_{\theta}^{z}
$$

(since $\mu_{\theta}^{0}=\mu_{\theta}$ we have $T^{0}=T$ ). Then the bound (1.3) will follow from Stein's complex interpolation theorem and the following mappings:

$$
\begin{array}{ll}
T^{-\frac{1}{2}+i \lambda}: L^{2}\left(\mathbb{R}^{2}\right) \rightarrow L^{2}\left(\mathbb{R}^{2} \times \mathbb{T}\right), & \lambda \in \mathbb{R}, \\
T^{1+i \lambda}: L^{1}\left(\mathbb{R}^{2}\right) \rightarrow L^{\infty}\left(\mathbb{R}^{2} \times \mathbb{T}\right), & \lambda \in \mathbb{R} .
\end{array}
$$

We prove (2.2) first.

By Lemma 1 and the computation of $\widehat{i_{z}}$ (see [GS]) we have

$$
\begin{aligned}
& \int_{-\pi}^{\pi} \int_{\mathbb{R}^{2}}\left|T^{-\frac{1}{2}+\lambda} f(x, \theta)\right|^{2} d x d \theta \\
& =\int_{\mathbb{R}^{2}} \int_{-\pi}^{\pi}|\widehat{f}(\xi)|^{2}\left|\widehat{\mu_{\theta}}(\xi)\right|^{2}\left|\widehat{i_{-\frac{1}{2}+i \lambda}}\left(\left(\sigma_{\theta} \xi\right) 2\right)\right|^{2} d \theta d \xi \\
& \leq c \int_{\mathbb{R}^{2}}|\widehat{f}(\xi)|^{2}|\xi| \int_{-\pi}^{\pi}\left|\widehat{\mu_{\theta}}(\xi)\right|^{2} d \theta d \xi \\
& \leq c \int_{\mathbb{R}^{2}}|\widehat{f}(\xi)|^{2} d x=c \int_{\mathbb{R}^{2}}|f(x)|^{2} d x .
\end{aligned}
$$

We now prove $(2.3)$.

It suffices to show that

$$
\left\|f * \mu_{\theta}^{1+i \lambda}\right\|_{L^{\infty}\left(\mathbb{R}^{2}\right)} \leq c\|f\|_{L^{1}\left(\mathbb{R}^{2}\right)}
$$

with a constant $c$ independent of $\theta$. It is enough to show that

$$
\left\|f_{-\theta} * \mu^{1+i \lambda}\right\|_{L^{\infty}\left(\mathbb{R}^{2}\right)} \leq c\|f\|_{L^{1}\left(\mathbb{R}^{2}\right)}
$$

where $\mu^{1+i \lambda}=\mu_{0}^{1+i \lambda}$ and therefore $\mu^{s}$ is defined by $\widehat{\mu^{s}}(\xi)=\widehat{\mu}(\xi) \widehat{i_{s}}\left(\xi_{2}\right)$. Since $\left\|f_{\theta}\right\|_{L^{1}\left(\mathbb{R}^{2}\right)}=\|f\|_{L^{1}\left(\mathbb{R}^{2}\right)}$ we have to show that $\mu^{1+i \lambda}$ is a function in $L^{\infty}\left(\mathbb{R}^{2}\right)$, i.e. that $\left|\left\langle\mu^{1+i \lambda}, g\right\rangle\right| \leq c$ for any function $g \in L^{1}\left(\mathbb{R}^{2}\right)$. Then, since $i_{1+i \lambda}$ acts only on the second coordinate,

$$
\begin{aligned}
\left|\left\langle\mu^{1+i \lambda}, g\right\rangle\right| & =\left|\int_{\mathbb{R}^{2}} \int_{\mathbb{R}^{2}} g(x+y) d \mu(x) i_{1+i \lambda}\left(y_{2}\right) d y\right| \\
& \leq \int_{\mathbb{R}} \int_{\mathbb{R}}\left|g\left(x_{1}, \phi\left(x_{1}\right)+y_{2}\right)\right| J\left(x_{1}\right) d x_{1} d y_{2} \leq c
\end{aligned}
$$

(the Jacobian $J$ is bounded if we have chosen the arc small enough; moreover $J$ is defined a.e. because of convexity). 
We now turn to the proof of Theorem 2, which depends on the following lemma (see $[$ BRT] $)$.

Lemma 2. Let $1<q \leq \infty$. If $\mu_{\theta}^{\gamma}$ is the measure defined in the statement of Theorem 2, then

$$
\left\{\int_{-\pi}^{\pi}\left|\widehat{\mu_{\theta}^{\gamma}}(\xi)\right|^{q} d \theta\right\}^{1 / q} \approx\left\{\begin{array}{lll}
|\xi|^{-1 / 2} & \text { for } & q<\frac{2 \gamma-2}{\gamma-2} \\
|\xi|^{-1 / 2}(\log |\xi|)^{\frac{\gamma-2}{2 \gamma-2}} & \text { for } & q=\frac{2 \gamma-2}{\gamma-2} \\
|\xi|^{-\frac{1}{q}-\frac{1}{\gamma}+\frac{1}{\gamma q}} & \text { for } & q>\frac{2 \gamma-2}{\gamma-2}
\end{array}\right.
$$

Proof of Theorem 2. Let $i_{z}$ be as in (2.1) and let $\mu_{\theta}^{\gamma, z}$ be defined by

$$
\widehat{\mu_{\theta}^{\gamma, z}}(\xi)=\widehat{\mu^{\gamma}}\left(\sigma_{\theta} \xi\right) \widehat{i_{z}}\left(\left(\sigma_{\theta} \xi\right)_{2}\right) .
$$

As in the previous proof we introduce the analytic family of operators

$$
T_{\gamma}^{z}: f \rightarrow f * \mu_{\theta}^{\gamma, z}(\xi)
$$

(again $T_{\gamma}^{0}: f \rightarrow f * \mu_{\theta}^{\gamma, z}(\xi)$ ).

Let $\alpha \geq 0$ and $q>2$. Suppose we have proved the following bounds:

$$
\begin{aligned}
T_{\gamma}^{-\alpha+i \lambda}: L^{2}\left(\mathbb{R}^{2}\right) \rightarrow L^{q}\left(\mathbb{T}, L^{2}\left(\mathbb{R}^{2}\right)\right), & \lambda \in \mathbb{R}, \\
T_{\gamma}^{1+i \lambda}: L^{1}\left(\mathbb{R}^{2}\right) \rightarrow L^{\infty}\left(\mathbb{T} \times \mathbb{R}^{2}\right), & \lambda \in \mathbb{R},
\end{aligned}
$$

then the complex interpolation theorem yields

$$
T_{\gamma}: L^{\frac{2(\alpha+1)}{2 \alpha+1}}\left(\mathbb{R}^{2}\right) \rightarrow L^{q(\alpha+1)}\left(\mathbb{T}, L^{2(\alpha+1)}\left(\mathbb{R}^{2}\right)\right) .
$$

The proof of (2.5) is similar to the proof of (2.3).

We now prove (2.4) for values of $q$ and $\alpha$ that will be made explicit in the course of the proof.

By Plancherel theorem and Minkowski's integral inequality we have

$$
\begin{aligned}
& \left\{\int_{-\pi}^{\pi}\left\{\int_{\mathbb{R}^{2}}\left|T_{\gamma}^{-\alpha+i \lambda} f(x)\right|^{2} d x\right\}^{q / 2} d \theta\right\}^{2 / q} \\
& =\left\{\int_{-\pi}^{\pi}\left\{\int_{\mathbb{R}^{2}}|\widehat{f}(\xi)|^{2}\left|\widehat{\mu_{\gamma}}\left(\sigma_{\theta} \xi\right)\right|^{2}\left|\left(i_{-\alpha+i \lambda}\right)^{\wedge}\left(\left(\sigma_{\theta} \xi\right)_{2}\right)\right|^{2} d \xi\right\}^{q / 2} d \theta\right\}^{2 / q} \\
& \leq \int_{\mathbb{R}^{2}}\left\{\int_{-\pi}^{\pi}\left(|\widehat{f}(\xi)|^{2}\left|\widehat{\mu_{\gamma}}\left(\sigma_{\theta} \xi\right)\right|^{2}\left|\left(i_{-\alpha+i \lambda}\right)^{\wedge}\left(\left(\sigma_{\theta} \xi\right)_{2}\right)\right|^{2}\right)^{q / 2} d \theta\right\}^{2 / q} d \xi \\
& \leq \int_{\mathbb{R}^{2}}|\widehat{f}(\xi)|^{2}|\xi|^{2 \alpha}\left\{\int_{-\pi}^{\pi}\left|\widehat{\mu_{\gamma}}\left(\sigma_{\theta} \xi\right)\right|^{q} d \theta\right\}^{2 / q} d \xi=I(q, \alpha) .
\end{aligned}
$$

By Lemma 2 we get

$$
I(q, \alpha) \leq c \int_{\mathbb{R}^{2}}|\widehat{f}(\xi)|^{2} d \xi=c \int_{\mathbb{R}^{2}}|f(x)|^{2} d x
$$

by either setting

$$
\alpha=1 / 2 \quad, \quad q<\frac{2(\gamma-1)}{\gamma-2}
$$


or

$$
\alpha=\frac{p^{\prime}-2}{2} \quad, \quad q=\frac{2(\gamma-1)}{\left(p^{\prime}-2\right) \gamma-2}
$$

(note that this forces $\alpha=\frac{1}{q}+\frac{1}{\gamma}-\frac{1}{\gamma q}$ ).

The first choice proves $(i)$ in the statement of Theorem 2, while the latter proves (ii) and $($ iii).

We now turn to the counterexamples. We prove $\left(i i^{\prime}\right)$, the proofs of the other cases being similar. We therefore assume

$$
T_{\gamma}: L^{p}\left(\mathbb{R}^{2}\right) \rightarrow L^{s}\left(\mathbb{T}, L^{p \prime}\left(\mathbb{R}^{2}\right)\right)
$$

and we shall deduce $s \leq \frac{p^{\prime}(\gamma-1)}{\left(p^{\prime}-2\right) \gamma-2}$.

Indeed, for small $\varepsilon>0$, let $f$ be the characteristic function of the rectangle $[-\varepsilon, \varepsilon] \times\left[-\varepsilon^{\gamma}, \varepsilon^{\gamma}\right]$. Then there exist constants $c, c_{1}, c_{2}, c_{3}$ such that

$$
f * \mu_{\theta}\left(x_{1}, x_{2}\right)>c \varepsilon
$$

for $\left|x_{1}\right|<c_{1} \varepsilon,\left|x_{2}\right|<c_{2} \varepsilon^{\gamma},|\theta|<c_{3} \varepsilon^{\gamma-1}$. Then (2.7) implies

$$
\left\{\int_{|\theta|<c_{3} \varepsilon^{\gamma-1}}\left\{\int_{\left|x_{1}\right|<c_{1} \varepsilon,\left|x_{2}\right|<c_{2} \varepsilon^{\gamma}} \varepsilon^{p^{\prime}} d x_{1} d x_{2}\right\}^{s / p^{\prime}} d \theta\right\}^{1 / s} \leq c \varepsilon^{\frac{1+\gamma}{p}},
$$

which yields $(\gamma+1)\left(\frac{1}{p}-\frac{1}{p^{\prime}}\right) \leq 1+\frac{\gamma-1}{s}$, i.e. $s \leq \frac{p^{\prime}(\gamma-1)}{\left(p^{\prime}-2\right) \gamma-2}$.

\section{REFERENCES}

[BIT] L. Brandolini, A. Iosevich and G. Travaglini, Spherical means and the restriction phenomenon, preprint.

[BRT] L. Brandolini, M. Rigoli and G. Travaglini, Average decay of Fourier transforms and geometry of convex sets, Rev. Mat. Iberoam. 14 (1998), 519-560. MR 2000a:42017

[C] M. Christ, Endpoint bounds for singular fractional integral operators, unpublished.

[GS] I.M. Gel'fand and G.E. Shilov, Generalized functions, Academic Press, 1964. MR 55:8786a

[O] D.M. Oberlin, Multilinear proofs for two theorems on circular averages, Coll. Math. LXIII (1992), 187-190. MR 93m:42005

[OS] D.M. Oberlin and E.M. Stein, Mapping properties of the Radon transform, Indiana Univ. Math. J. 31 (1982), 641-650. MR 84a:44002

[P] A.N. Podkorytov, The asymptotics of a Fourier transform on a convex curve, Vestn. Leningr. Univ. Mat. 24 (1991), 57-65. MR 93h:42019

[Ra] B. Randol, A lattice point problem, Trans. Amer. math. Soc. 121 (1966), 257-268. MR 34:1291

[Ri] F. Ricci, $L^{p}-L^{q}$ boundedness for convolution operators defined by singular measures in $\mathbb{R}^{n}$, Boll. Un. Mat. Ital. A 11 (1997), 237-252. MR 99c:42023

[RS] F. Ricci and E.M. Stein, Harmonic analysis on nilpotent groups and singular integrals. III. Fractional integration along manifolds, Journ. Funct. Anal. 86 (1989), 360-389. MR 90m:22027

[S] R. Strichartz, Convolution with kernels having singularities on a sphere, Trans. Amer. Math. Soc. 148 (1970), 461-471. MR 41:876

Dipartimento di Matematica, Politecnico di Torino, Corso Duca degli Abruzzi 24, 10129 TORINO, ITALY

E-mail address: fricci@polito.it

Dipartimento di Matematica e Applicazioni, Università di Milano Bicocca, Via BicocCA DEGLi ARCimboldi 8, 20126 Milano, Italy

E-mail address: travaglini@matapp.unimib.it 